\title{
« Je suis heureux d'avoir l'âge que j'ai » : la résilience des hommes gais âgés vivant avec le VIH au Québec
}

\author{
Isabelle Wallach \\ Université du Québec à Montréal
}

\begin{abstract}
RÉSUMÉ
La présente recherche vise à mettre en lumière la résilience des hommes gais âgés vivant avec le VIH, acquise à travers leur parcours relié à l'homosexualité, au VIH et au vieillissement. S'inscrivant dans une approche qualitative, cette recherche s'appuie sur des entrevues semi-dirigées réalisées avec 10 hommes gais vivant avec le VIH âgés de 50 à 71 ans. L'analyse des données a permis de mettre en évidence 7 thématiques reflétant la résilience de cette population : la maturité, l'acceptation de soi, les épreuves surmontées, l'autonomie, l'appréciation de la valeur de la vie, l'attitude positive et le sentiment d'être chanceux.
\end{abstract}

Mots clés : Hommes, homosexualité, VIH, vieillissement, résilience, méthodologie qualitative

Souvent associée à la population jeune dans les représentations populaires, l'infection à VIH touche en réalité une proportion de plus en plus importante de personnes vieillissantes. Ce phénomène s'expliquerait à la fois par la chronicisation de l'infection suite à l'apparition de thérapies antirétrovirales efficaces, qui permettent désormais aux individus de vieillir avec le VIH, et par l'augmentation de la proportion des nouvelles infections chez les personnes de 50 ans et plus. Au Canada, entre 1999 et 2008, la proportion des nouveaux cas de VIH concernant les personnes de 50 ans et plus est passée de 10,6 à 15,3\%, et celle des cas de sida, de 15,9 à 21,6\% (Agence de la santé publique du Canada, 2010). Alors que les hommes gais ${ }^{1}$ représentent

Isabelle Wallach, professeure, département de sexologie, Université du Québec à Montréal et chercheure associée au Centre de recherche et d'expertise en gérontologie sociale, CSSS Cavendish.

Cette recherche a été soutenue par un financement SIRA (Soutien aux initiatives visant au respect des aînés) du ministère de la Famille et des Aînés du Québec octroyé à la Fondation l'Actuel. L'auteure remercie Xuân Ducandas et Michel Martel pour leur assistance dans la codification et la réalisation des entrevues.

La correspondance concernant cet article devrait être acheminée à Isaballe Wallach, professeure, département de sexologie, Université du Québec à Montréal, Case postale 8888, succursale Centre-ville, Montréal (Québec) H3C 3P8. Tél : 514-987-3000, poste 1645. Téléc : 514-987-6787. Courriel : wallach.isabelle@uqam.ca 
le groupe le plus anciennement affecté par l'infection à VIH, ils demeurent encore aujourd'hui celui le plus largement touché parmi la population des personnes vivant avec le VIH de 50 ans et plus. Ainsi, entre 1985 et 2008, les hommes ayant des relations sexuelles avec les hommes (HARSAH) comptaient pour 48,7\% des nouveaux cas de VIH et pour $61,3 \%$ des diagnostics de sida au sein du groupe des personnes de 50 ans et plus vivant avec le VIH (Agence de la santé publique du Canada, 2010).

Les travaux portant sur les personnes de 50 ans et plus vivant avec le VIH (PVVIH50+) soulignent les nombreuses difficultés d'ordre psychosocial et socioéconomique auxquelles cette population est confrontée : vieillissement physique prématuré (Desquilbet et al., 2007 ; Effros et al., 2008), troubles de santé et comorbidités (Havlik, 2009 ; Power, Bell et Freemantle, 2010), dégradation de l'estime de soi (Nichols et al., 2002), peur de mourir (Vance et Woodley, 2005), stigmatisation du VIH et âgisme (Emlet, 2006a); isolement (Emlet, 2006b ; Poindexter et Shippy, 2008), situation de non-emploi et difficultés économiques (Power et al., 2010), troubles de santé mentale (Applebaum et Brennan, 2009 ; Kalichman, Heckman, Kochman et Bergholte, 2000). Peu de recherches ont porté spécifiquement sur la population des hommes gais âgés vivant avec le VIH. Une recherche qualitative (Linsk, 1997) relève cependant certaines difficultés spécifiques ou plus prononcées chez cette population, telles que l'acceptation du vieillissement, les inquiétudes relatives à l'évolution de la santé ainsi que l'âgisme qui touche particulièrement les hommes gais âgés. Selon Lyons, Pitts, Grierson, Thorpe et Power (2010), les gais âgés vivant avec le VIH seraient confrontés à des défis uniques en raison de la triple stigmatisation relative à leur statut VIH, leur âge et leur orientation sexuelle.

Malgré ces multiples difficultés, quelques recherches ont pointé des forces et des processus de résilience caractérisant les PVVIH50+, notamment la sagesse associée au vieillissement, un plus grand respect de la vie et de la santé, une plus grande patience et un sens de la satisfaction (Siegel, Raveis et Karus, 1998), une vision positive du vieillissement (Vance et Woodley, 2005), l'acceptation de soi, l'optimisme, le désir de vivre, la générativité et l'indépendance (Emlet, Tozay et Raveis, 2010). Bien qu'aucune des recherches sur les forces et la résilience des PVVIH50+ ne porte spécifiquement sur la population des hommes gais âgés, la littérature générale sur les personnes homosexuelles âgées nous donne des indices concernant la résilience de cette population. De nombreux travaux soulignent en effet que la « crise de compétence » vécue à travers le processus d'acceptation de leur identité sexuelle et du coming out permet à ces personnes d'acquérir des habiletés de coping qui les préparent à la gestion de la stigmatisation et des pertes associées au vieillissement (Butler, 2004 ; Friedriksen-Goldsen et Muraco, 2010 ; Genke, 2004 ; Morrow, 2001). Les rejets de la famille auraient aussi amené les personnes lesbiennes, gaies, bisexuelles et transsexuelles/transgenres (LGBT) à développer une forme d'indépendance pouvant être bénéfique dans l'étape du vieillissement (Morrow, 2001).

La question que nous nous proposons de traiter dans cet article est celle de savoir si, en dépit des multiples défis soulevés par l'intersection de l'homosexualité, du vieillissement et du VIH, les hommes gais âgés de 50 ans et plus vivant avec le VIH témoignent d'attitudes ou de comportements qui relèvent du phénomène de la résilience. De plus, nous chercherons à examiner si la résilience acquise à travers le parcours relié à l'homosexualité, au VIH et au vieillissement peut aider ces hommes gais âgés à mieux faire face aux difficultés associées au VIH et au vieillissement.

Dans le cadre de cette recherche, la résilience sera définie comme un "patron de fonctionnement indiquant une adaptation positive dans le contexte de risque significatif ou d'adversité $»^{2}$ (Ong, Bergeman et Boker, 2009, p. 1777). Selon Ong et ses collègues, le terme de risque réfère ici à des événements de vie négatifs, des 
traumas ou des difficultés de vie chronique tandis que le terme d'adaptation positive désigne une adaptation substantiellement meilleure que ce qui pourrait être attendu compte tenu de l'exposition au risque. Nous privilégierons une perspective qui considère la résilience comme un processus dynamique (Hildon, Smith, Netuveli et Blane, 2008 ; Luthar, Cicchetti et Becker, 2000 ; Michallet, 2009/2010) plutôt que comme reliée à des traits inhérents à la personne. Ainsi, nous nous intéresserons particulièrement aux forces acquises par les personnes dans l'adversité, notamment sous l'influence des défis imposés par l'homosexualité, par le VIH et par le vieillissement. De plus, nous porterons notre attention sur les mécanismes de coping, c'està-dire les processus, pensées ou comportements mis en place par les individus pour gérer les facteurs de stress (Greve et Staudinger, 2006) qui, pour certains auteurs, sont considérés comme une facette importante du processus de résilience (Beasley, Thompson et Davidson, 2003 ; Greve et Staudinger, 2006). Bien que la résilience soit aussi fortement influencée par la présence de ressources externes comme les relations sociales avec l'entourage ou le recours à la spiritualité (Hildon et al., 2008 ; Pentz, 2005), dans cet article nous nous centrerons exclusivement sur les ressources internes des personnes, à savoir sur les forces acquises ou les stratégies de coping s'appuyant sur l'individu lui-même.

\section{MÉTHODOLOGIE}

Cette recherche privilégie une approche qualitative qui met l'accent sur les processus et les significations, ainsi que sur la compréhension approfondie de la perspective des acteurs (Denzin et Lincoln, 1994). Les données présentées s'inscrivent dans un projet plus large portant sur l'expérience et les difficultés des PVVIH50+ qui inclut différentes populations. Seuls les résultats portant sur les HARSAH (non utilisateurs de drogues par injection) seront présentés ici.

\section{Recrutement et échantillon}

Les participantes et participants à la recherche ont été recrutés au sein de la Clinique médicale l'Actuel, située à Montréal dans la province du Québec. Le recrutement s'est fait par l'intermédiaire des médecins afin de respecter la confidentialité concernant le statut infectieux des participants et participantes.

Les critères de recrutement des participantes et participants étaient qu'ils soient âgés de 50 ans et plus et qu'ils vivent avec le VIH. L'échantillon a été diversifié concernant l'âge des individus, l'âge du diagnostic (avant et après 50 ans) et l'ancienneté du diagnostic. Le recrutement des participants et participantes a été arrêté quand la saturation théorique a été atteinte, les discours des participants et participantes ne venant plus ajouter de données nouvelles qui contredisent les catégories d'analyse identifiées.

L'échantillon de l'ensemble du projet se compose de 38 participants et participantes. Il inclut des hommes et des femmes hétérosexuels caucasiens, des hommes et des femmes hétérosexuels de minorités ethniques, des hommes (hétérosexuels et gais) et des femmes usagers de drogues par injection, ainsi que des hommes ayant des relations sexuelles avec des hommes (HARSAH) caucasiens non utilisateurs de drogues par injection. Le fait de ne pas avoir inclus d'HARSAH de minorités ethniques dans l'échantillon n'est pas un choix mais un biais relié au lieu du recrutement.

Les résultats présentés ici se centreront sur la population des HARSAH caucasiens et infectés par voie sexuelle, l'usage de drogue par injection constituant une problématique très spécifique qui mérite d'être 
traitée séparément. L'échantillon du groupe des HARSAH compte 10 hommes âgés de 50 à 71 ans. Un seul participant se définit actuellement comme bisexuel, mais trois autres ont eu des relations durables dans le passé avec des femmes. Deux des participants ont des enfants adultes et l'un des deux est également grandpère. Six sont dans la cinquantaine et quatre dans la soixantaine ou plus. Deux hommes ont été récemment diagnostiqués ( 2 ans et 4 ans avant l'entrevue), trois ont vécu entre 10 et 15 ans avec l'infection à VIH et cinq ont été diagnostiqués il y a 20 ans ou plus. Tous sont des Québécois parlant français.

Sur le plan économique, deux participants sont actuellement en situation d'emploi, trois sont à la retraite et quatre ont cessé leur activité professionnelle et perçoivent des prestations sociales (aide sociale ou rente d'invalidité). Quatre participants reçoivent un revenu annuel de plus de 40000 \$ et six vivent avec moins de 20000 \$ par an, c'est-à-dire en dessous du seuil de faible revenu établi par le gouvernement du Canada, lequel est considéré au Québec comme le seuil de pauvreté.

\section{Collecte et analyse des données}

La recherche s'appuie sur des entrevues individuelles semi-dirigées approfondies d'une durée moyenne de 2 heures 30. Les entrevues ont été réalisées dans les locaux de la clinique à l'exception d'une, faite au domicile d'un participant. Tous les participants ont été informés des objectifs de la recherche et invités à signer un formulaire de consentement avant le début de l'entrevue. Les précautions habituellement prises pour respecter les principes éthiques de la recherche ont été suivies, en particulier concernant le bien-être du participant, son droit à ne pas répondre aux questions et à se retirer en tout temps du projet, ainsi que le respect de la confidentialité et de l'anonymat. Une somme de $40 \$$ a été remise à tous les participants à la fin de l'entrevue à titre de compensation symbolique.

Les entrevues s'organisent autour de cinq grandes thématiques : le diagnostic et le vécu du VIH, l'expérience du vieillissement, la vie personnelle et sociale, les ressources internes et informelles des personnes pour faire face à leurs difficultés et l'expérience des ressources formelles. La question des répercussions du VIH et du vieillissement sur l'existence de la personne traverse l'ensemble des thèmes abordés. L'entrevue se clôt par la passation d'un court questionnaire visant à recueillir des données sociodémographiques sur le participant.

Toutes les entrevues ont été intégralement retranscrites. L'analyse des données s'est basée sur l'approche de la théorisation ancrée (Strauss et Corbin, 1998), s'appuyant sur un processus de comparaison constante entre les données d'un côté et les codes et catégories créés de l'autre. Dans un premier temps, la lecture des premières entrevues et leur codification ouverte sur papier nous a permis d'établir une première liste de catégories et de codes à partir des thématiques récurrentes identifiées dans les discours mais aussi de thèmes issus de la grille d'entrevue ou de la littérature. Cette liste de codes et de catégories a ensuite été utilisée pour procéder à une première codification des entrevues à l'aide du logiciel QDA Miner. La codification de chaque entrevue a été réalisée par deux membres de l'équipe de recherche afin de garantir la triangulation. Un travail de relecture régulière du matériel associé à chaque code a permis de raffiner nos codes et de les redéfinir, ainsi que d'en générer de nouveaux à mesure que de nouvelles entrevues étaient codifiées. Il nous a ainsi été possible d'identifier une catégorie résilience à partir de codes se trouvant initialement dans d'autres catégories, tels que forces, attitude face aux difficultés, façon d'être et philosophie de vie, rapport à la mort, gestion de la stigmatisation. Nous avons ensuite procédé au codage axial, en définissant de façon 
plus précise les sous-catégories de la catégorie résilience. Durant cette étape, nous avons notamment comparé les expériences des différents sous-groupes de populations inclus dans notre échantillon afin de mieux saisir les variations des différentes sous-catégories en fonction de variables telles que le genre et l'orientation sexuelle. L'étape de la codification sélective et de la théorisation de l'ensemble des données du projet n'a pas encore été réalisée.

\section{RÉSULTATS}

La comparaison intergroupe des données nous a permis d'identifier les facettes de la résilience qui concernaient plus spécifiquement les participants HARSAH. Les thèmes présentés ici sont ceux qui sont apparus plus caractéristiques des participants HARSAH que des femmes ou des hommes hétérosexuels. Sept grandes thématiques reflétant la résilience des hommes gais âgés vivant avec le VIH ont ainsi émergé de l'analyse: la maturité, l'acceptation de soi, les épreuves surmontées, l'autonomie, l'appréciation de la valeur de la vie présente, l'attitude positive et le sentiment d'être chanceux.

\section{La maturité}

La maturité a été décrite par plusieurs participants comme la principale force associée au vieillissement dont ils peuvent percevoir les effets. La maturité est associée à la sagesse et l'expérience, et souvent mise en parallèle avec l'inexpérience qui caractérise la jeunesse. Alors que le vieillissement est perçu par beaucoup

comme une étape difficile à vivre, la sagesse qui l'accompagne lui confère une valeur positive et contribue à son acceptation :

Souvent, je suis heureux d'avoir l'âge que j'ai. Pis je ne voudrais pas avoir mes 20 ans, parce que je sens plus de sagesse, plus de chemin de fait, plus de parcours. (H55)

La maturité est également considérée comme un atout pour faire face aux multiples difficultés de l'existence. D'après le discours des participants, elle les aiderait à prendre de la distance par rapport aux événements perturbants de la vie. Un des participants souligne l'évolution de sa façon de gérer les difficultés de l'existence qui se traduit par une tranquillité d'esprit et un plus grand contrôle de ses émotions :

On passe par-dessus les petites affaires, t'sais ... On s'inquiète moins mettons. Puis avant je me faisais des montagnes t'sais pour des riens, mais astheure non, comme je disais, on devient plus serein. (H50)

Cette sagesse peut s'avérer jouer un rôle essentiel dans l'expérience du VIH. Ainsi, le participant ayant été infecté récemment, après l'âge de 50 ans, estime que la maturité associée à son âge a favorisé l'acceptation de l'infection à VIH :

Si je l'avais eu quand j'avais 30 ans, comment j'aurais réagi versus à la façon dont j'ai réagi 5 ans passés, ça aurait été complètement différent, là. À 30 ans, le côté sagesse est probablement moins développé qu'à 50. (H56)

\section{L'acceptation de soi}

Le fait de s'accepter soi-même est une autre force qui ressort des discours des répondants HARSAH alors qu'il est totalement absent de ceux des femmes et des hommes hétérosexuels. Quatre des participants 
gais ont fait référence à une acceptation de soi, se manifestant notamment à travers l'acceptation des aspects de leur identité stigmatisés par la société comme le VIH, l'homosexualité ou le vieillissement.

L'acceptation de soi peut par exemple se refléter dans l'acceptation des changements corporels reliés au vieillissement. Cette acceptation de son propre vieillissement semble être à la source d'une stratégie de coping consistant à prendre de la distance par rapport aux expériences de rejet âgistes courantes au sein de la communauté gaie, en particulier dans le contexte des rapports de séduction. Un des participants ayant été l'objet de comportements âgistes dans un sauna en raison des signes physiques de son vieillissement, rapporte ainsi ne pas avoir été affecté par ces comportements stigmatisants grâce à sa propre acceptation de soi :

Ben regarde, s'ils ne m'aiment pas comme je suis, qu'est-ce que tu veux que je fasse ? Je peux pas rien y faire. Alors moi, je n'ai pas le choix de m'accepter puis si eux, ils ne m'acceptent pas, bien qu'ils passent. Puis qu'ils aillent à l'autre chambre. [rire] (H50)

Pour un des répondants, cette meilleure acceptation de soi semble avoir débuté avec le processus d'acceptation de son homosexualité. Selon ses propos, la décision d'assumer pleinement cette partie de son identité l'a aidé par la suite à accepter également son identité de personne vivant avec le VIH :

Je pense que ça vient d'un jour avoir accepté mon homosexualité aussi. À un moment donné, tu décides que oui, j'accepte, c'est fini de me cacher, je retourne plus en arrière. [ . . . ] Je pense, c'est ce qui m'aide peut-être à passer au travers puis à en parler plus facilement [du VIH]. (H52)

L'acceptation de soi semble aussi être renforcée par le processus de vieillissement. Alors qu'un des participants explique que les traits de personnalité qui étaient sources de malaise durant la jeunesse finissent par être acceptés en vieillissant, celui qui avait évoqué le rôle de l'homosexualité dans l'acceptation du VIH fait référence au rôle majeur du vieillissement dans le déploiement d'une acceptation de soi totale :

C'est en vieillissant que j'ai appris à m'aimer plus. [ . . . ] Accepter le VIH, ça fait partie de t'accepter toi, d'accepter tes bons puis tes mauvais côtés aussi. [ . . . ] J'accepte mieux mon homosexualité, ma vie, t'sais tout! En vieillissant, j'accepte ce que je suis, point. (H52)

\section{Les épreuves surmontées}

Le thème des épreuves surmontées s'est avéré commun aux femmes et aux HARSAH. En revanche, il est très peu présent dans le discours des hommes hétérosexuels. La question de l'ancienneté du diagnostic et donc du parcours relié au VIH joue sans doute un rôle important dans l'apparition de cette force acquise, car les participants hommes hétérosexuels ont en moyenne vécu moins longtemps avec le VIH que les participants femmes et HARSAH. Avoir vécu avec le VIH, avoir développé des maladies graves mais aussi

avoir dû assumer son homosexualité, sont autant d'événements biographiques qui peuvent avoir représenté des épreuves pour les gais âgés vivant avec le VIH. De façon paradoxale, ces épreuves, une fois dépassées, semblent avoir conféré une force à certains participants HARSAH et les avoir rendus plus résistants face aux nouvelles difficultés rencontrées.

Deux participants considèrent que le chemin parcouru par rapport au VIH leur a permis de se renforcer en leur montrant qu'ils étaient capables de faire face à l'adversité. Pour le second répondant, outre le VIH, c'est son coming out réalisé alors qu'il était marié et père de famille ainsi que la rupture qui s'en est suivie 


\section{LA RÉSILIENCE DES HOMMES GAIS ÂGÉS VIVANT AVEC LE VIH AU QUÉBEC}

dans son couple et avec ses enfants qui constituent des épreuves majeures de l'existence ayant contribué à le renforcer :

Je regarde en arrière pis là, je me dis : «T'as eu ton premier enfant, t'as caché ton homosexualité jusqu'à 40 ans . . . \ J'ai passé à travers mon homosexualité, j'ai passé à travers un divorce. [ . . . ] Là, j'ai eu un cancer. Ma vie, elle continue encore. [ . . . ] Si on n'a pas d'épreuves, on peut pas avancer. (H62)

Deux autres participants ont par ailleurs rapporté avoir le sentiment que l'expérience d'avoir surmonté des épreuves difficiles les rendait beaucoup plus aguerris que des personnes de leur entourage face aux problèmes de l'existence. L'un des deux qui a été atteint de plusieurs cancers, dont certains sont reliés au $\mathrm{VIH}$, et qui a également connu des expériences de rejet de la part de certains membres de sa famille lorsqu'il a fait son coming out après 23 ans de mariage, fait ce constat :

Des fois, je me sens que j'ai plus de résistance que d'autres ont, parce que je regarde mes amis à côté, des fois, y ont juste une petite affaire pis y sont démoralisés. (H65)

\section{L'autonomie}

Le thème de l'autonomie a émergé de l'analyse des discours des répondants à travers plusieurs dimensions, à savoir la volonté de gérer les difficultés pratiques et morales de façon autonome et l'autonomie émotionnelle. Sept des dix participants HARSAH ont évoqué l'une ou l'autre de ces facettes de l'autonomie.

Quatre répondants ont évoqué préférer régler leurs difficultés pratiques de façon indépendante en évitant de solliciter l'aide de leur entourage, quelle que soit la nature de leur problème (santé, difficultés financières, perte d'autonomie fonctionnelle). Un des participants ayant été confronté à de graves difficultés financières raconte :

J'ai jamais été quelqu'un qui a été capable de demander. T'sais je m'arrange avec ce que j'ai. La famille,

c'est eux autres qui sont venus. C'est pas moi qui les ai appelés pour demander de l'aide. (H52)

De façon semblable, plusieurs discours attestent de la volonté des participants HARSAH de préserver leur autonomie même en cas de limitations fonctionnelles dues à la maladie ou à un handicap physique, comme le montre cette citation :

Même cet hiver, j'étais bien malade, on a voulu faire le ménage chez moi, j’ai dit : « Non. Tant que je vais être capable, je vais le faire ». (H65)

L'importance de l'autonomie pour les participants HARSAH transparaît également dans leur propos concernant leur volonté de gérer seul les difficultés morales auxquelles ils peuvent être exposés. Plusieurs rapportent ainsi préférer ne pas se confier à leur entourage en cas de problème ou ne pas demander de soutien à leurs proches et faire face seuls aux problèmes :

Mettons que ça va mal, il y en a qui se mettent à pleurer puis ils appellent leur chum. Je n'ai jamais eu besoin de ça. J'aime pas que personne sache mes problèmes. (H71)

Enfin, pour certains participants, cette volonté d'être autonome sur le plan émotionnel se traduit par une tendance à apprécier la solitude, comme le montrent ces propos :

Je suis heureux avec moi-même. J'écoute les autres mais je ne me confie pas aux autres. (H63) 
Un des répondants décrit sa sérénité face à la solitude et l'acquisition d'une autonomie émotionnelle comme le fruit d'un cheminement en lien avec son parcours relatif au VIH, qu'il a pu accomplir grâce à l'aide de son partenaire, d'un travailleur social et de son médecin :

Ce que j'ai le plus appris, je te dirais, ce que j'étais pas capable de faire avant, c'était la solitude. [... ] Quand tu penses que tu as besoin de tout le monde pour être heureux, un amant à tout prix, de l'argent à tout prix ... y a quelque chose qui va pas. Pis j'étais comme ça. Puis aujourd'hui, je suis bien dans ma solitude. (H62)

\section{L'appréciation de la valeur de la vie présente}

Les discours des répondants mettent en évidence deux attitudes qui semblent étroitement interreliées : la conscience de la proximité de la mort et une appréciation de la valeur de la vie et de l'instant présent. La conscience de la finitude qui s'enracine d'une part, dans l'expérience de l'infection à VIH longtemps perçue comme une maladie létale, et d'autre part, dans le vieillissement associé à une plus grande proximité de la mort, semblerait être à l' origine d'une plus grande valeur accordée à l'existence par les participants HARSAH.

Témoignant d'une conscience aigüe de la valeur de la vie, plusieurs répondants ont rapporté vivre « au jour le jour » et apprécier le moment présent. En adoptant cette philosophie de vie, les personnes semblent se libérer de l'anxiété qui pourrait naître de l'incertitude concernant l'avenir. Cette stratégie parait particulièrement efficace pour faire face aux inquiétudes reliées au risque de maladie, comme l'illustre cet extrait d'entrevue :

Je prends ça au jour le jour, je ne m'inquiète pas [de] ce qui peut arriver, si les pilules font défaut, ben elles feront défaut. Si je tombe malade, ben je tomberai malade. Je le prends comme ça. (H71)

L'appréciation de la valeur de la vie semble aussi générer, chez certains participants HARSAH, une attitude de détachement qui consiste à réduire ses attentes ou à mieux accepter de ne pas les voir se réaliser. Cette attitude de détachement se fait jour plus particulièrement dans la sphère intime, la valeur de la vie paraissant venir compenser la solitude amoureuse vécue par certains participants. Ainsi, deux d'entre eux affirment dans le même temps aspirer à construire une nouvelle relation intime et être prêt à accepter que leur vœu ne se réalise pas :

À mon âge, t'sais, les chances sont moins grandes que si j'avais 25 ou 30. Mais l'importance est aussi moins grande. En autant que je vis bien, pis que ma santé est bonne, je pense que la vie est belle. (H56)

\section{Une attitude positive}

Une des autres forces identifiées chez les participants HARSAH a trait à leur attitude positive face à la vie, qui se manifeste également à travers leur optimisme et leur espoir. L'origine de cette attitude est rarement formulée, mais elle est présente chez sept d'entre eux.

La croyance en sa propre capacité à faire face aux épreuves est une des déclinaisons de cette attitude positive. Un participant affirme :

Ça a toujours été ma façon d'aller chercher mon énergie, me dire qu'il y allait avoir un bon moment qui allait venir où je penserai plus à ça pis y faut pas que je m'en fasse. (H53)

Ayant survécu à trois cancers, un autre s'exprime en des termes semblables : 


\section{LA RÉSILIENCE DES HOMMES GAIS ÂGÉS VIVANT AVEC LE VIH AU QUÉBEC}

J'aurais pas cette croyance-là d'aller chercher, puiser une force en moi pour me dire : « Regarde, là, tu vas t'en sortir » puis d'y croire, je serais pas là aujourd'hui ». (H65)

Le désir de conserver un moral positif représente une autre facette de cette attitude. Deux répondants ont décrit cette volonté de garder leur bonne humeur au lieu de se laisser envahir par des émotions négatives. Notons que le maintien d'un moral positif participe ici d'une démarche intentionnelle et d'un véritable travail sur soi, comme on peut le percevoir dans ces propos :

C'est sûr qu'il y a des journées t'es moins en forme que d'autres ... Je me parle mentalement, t'sais ... Si ça ne va pas ben, j'essaie de faire autre chose pour que ça aille mieux. [ . . . ] Puis en disant que ça va ben, ben c'est sûr que ça aide aussi. Si tu dis toujours que ça va mal, ben tu déprimes. (H52)

\section{Le sentiment d'être chanceux}

Tous les participants HARSAH dont le discours témoigne d'une attitude positive évoquent, à certains moments de leur discours, le sentiment d'être " chanceux » ou " gâtés ». Ce sentiment concerne le plus souvent la santé, mais il peut aussi s'appliquer à d'autres dimensions comme l'apparence physique encore jeune, la vie amoureuse passée, le soutien social reçu, la situation matérielle, le logement, la vie professionnelle ou le parcours de vie en général.

Le sentiment d'être chanceux peut être considéré comme un mécanisme de coping qui permet de relativiser le caractère négatif de sa condition comparativement à ce qui serait attendu. Ce sentiment peut notamment se construire dans la comparaison avec d'autres personnes avec lesquelles le participant partage une caractéristique commune, telle que l'âge ou le VIH. Se percevoir comme chanceux signifie d'une certaine façon tirer un bilan positif de la situation passée et présente, au regard des embûches rencontrées dans le parcours de vie.

Plusieurs participants ont par exemple rapporté apprécier leur bonne condition de santé physique au regard des multiples épisodes de maladie vécus par des personnes vivant avec le VIH dans leur entourage. Pour l'un d'entre eux, c'est son expérience comme bénévole dans un organisme communautaire de lutte contre le VIH qui l'a amené à relativiser sa situation :

On regarde les autres puis on se trouve chanceux. Il y en a qui sont maganés, ça n’a pas d'allure. (H50)

Plus généralement, le fait d'être encore en vie et d'avoir survécu au VIH contribue largement, pour certains, à ce sentiment d'être chanceux. Cette posture peut favoriser l'acceptation du vieillissement, comme le montre cet extrait d'entrevue :

En vieillissement, tout est beau. [ ... ] Astheure, je me dis : «Bon, ben, je suis ben chanceux d'être debout à matin. [ . . . ] Je suis ben chanceux d'être ben vivant toujours en ayant le VIH ». (H62)

\section{DISCUSSION}

Les résultats de cette recherche ont mis en évidence plusieurs forces et mécanismes de coping qui attestent de l'existence d'une résilience chez les gais âgés de 50 ans et plus vivant avec le VIH. Les formes de résilience identifiées dans notre étude recoupent en partie celles décrites dans les recherches portant sur 
les PVVIH50+, mais présentent aussi des spécificités qui font écho aux travaux portant sur la population homosexuelle âgée.

La maturité associée à l'avancement en âge a été évoquée par la plupart des participants comme une force les aidant à mieux faire face aux difficultés de l'existence, à prendre du recul et à tempérer leurs réactions, ce qui rejoint les résultats de Siegel et ses collègues (1998) relatifs au thème de la sagesse. D'après nos données, la maturité pourrait, en outre, faciliter l'acceptation de l'infection par le VIH pour les personnes récemment diagnostiquées. Bien que l'avantage de recevoir le diagnostic du VIH en étant âgé soit mentionné par d'autres travaux (Linsk, 1997 ; Siegel et al., 1998), ceux-ci n'établissent pas de relation entre la sagesse associée au vieillissement et cette acceptation du VIH. L'originalité de nos résultats réside aussi dans la mise en évidence que la maturité est perçue par les répondants comme un aspect bénéfique du vieillissement et contribue de ce fait à son acceptation. Cet aspect paraît d'autant plus important pour des HARSAH âgés qui doivent faire face à une dévalorisation liée au vieillissement et à un âgisme fortement prégnant au sein de la communauté gaie. Le fait que la maturité soit davantage soulignée par les participants HARSAH que par les femmes et les hommes hétérosexuels pourrait d'ailleurs s'expliquer par un besoin plus impérieux de valoriser les effets bénéfiques du vieillissement compte tenu de la difficulté plus grande à l'accepter.

L'acceptation de soi constitue une autre manifestation de la résilience des hommes gais âgés vivant avec le VIH, que l'on retrouve au sein de la population générale des PVVIH50+ (Emlet et al., 2010). Dans le cas des HARSAH, l'acceptation de soi pourrait ici encore agir comme un moyen de coping important pour faire face aux comportements âgistes caractéristiques de la communauté gaie (Herdt et de Vries, 2004 ; Schope, 2005). Nos résultats ont par ailleurs permis de mettre au jour le rôle joué, d'une part, par le vieillissement et, d'autre part, par l'acceptation de l'homosexualité dans le développement de cette acceptation de soi. L'influence du vieillissement dans le renforcement de l'acceptation de l'orientation sexuelle des hommes gais âgés a déjà été mise en évidence par une recherche sur les Afro-américains gais et bisexuels (Della, Wilson et Miller, 2002). Par ailleurs, les travaux sur les LGBT âgés (Butler, 2004 ; Friedriksen-Goldsen et Muraco, 2010 ; Morrow, 2001) démontrent que cette population développerait durant sa jeunesse des aptitudes de coping à travers le processus d'acceptation d'une orientation sexuelle stigmatisée par la société qui pourrait être réutilisées ultérieurement dans l'acceptation d'autres identités stigmatisées, telles que celle de personne vieillissante ou vivant avec le VIH. L'intersection du vieillissement et de l'homosexualité semblerait donc particulièrement propice à favoriser une acceptation de soi chez les hommes gais de 50 ans et plus vivant avec le VIH.

L'autonomie apparaît comme une autre force de certains hommes gais âgés vivant avec le VIH, corroborant les résultats d'Emlet et ses collègues (2010) concernant l'autogestion des PVVIH50+. Certains de nos répondants partagent avec les autres PVVIH50+ une volonté de résoudre par eux-mêmes leurs difficultés, aussi bien sur le plan pratique que personnel. On peut cependant se demander dans quelle mesure cette volonté de gestion autonome de son existence ne s'enracine pas, pour la population gaie, dans le parcours relatif à l'homosexualité puisque, comme le soulève Morrow (2001), l'homophobie aurait conduit les personnes homosexuelles à développer une forte indépendance qui se maintiendrait au moment du vieillissement. La dimension émotionnelle de l'autonomie dont font preuve certains des participants HARSAH pourrait aussi s'enraciner dans cette indépendance acquise à travers le parcours relié à l'homosexualité. Favorisant un vécu positif de la solitude, cette autonomie émotionnelle parait particulièrement importante compte tenu 
de la fragilité des réseaux sociaux et du risque d'isolement des PVVIH50+ (Poindexter et Shippy, 2008 ; Shippy et Karpiak, 2005).

L'appréciation de la valeur de la vie présente s'est également révélée comme une force des hommes gais âgés vivant avec le VIH que la conscience de la fragilité de l'existence conduit à développer une philosophie de vie centrée sur l'instantanéité. Nos données ont fait émerger la relation existant entre cette appréciation de la valeur de la vie et le détachement dont font preuve certains HARSAH âgés par rapport à la réalisation de leurs aspirations, notamment dans la vie amoureuse et sexuelle. Nos résultats permettent donc d'établir un lien entre les thèmes du sens de la satisfaction (Siegel et al. ,1998) et du désir de vivre (Emlet et al., 2010) relevés à propos de la population des PVVIH50+, lesquels paraissent également interreliés avec la conscience de la proximité de la mort caractéristique de l'intersection du VIH et du vieillissement.

Le thème des épreuves surmontées est également apparu comme central dans le discours de plusieurs participants. Si le VIH figure au nombre de ces épreuves, nos résultats montrent que dans le cas des gais âgés, la gestion de la crise associée au coming out peut aussi être comptée comme un des événements difficiles ayant contribué à renforcer les individus, corroborant les analyses sur les forces acquises par les gais âgés (Butler, 2004 ; Morrow, 2001).

Pour finir, nos résultats ont mis au jour deux thèmes connexes qui sont l'attitude positive et le sentiment d'être chanceux. Ces deux thèmes dénotent un regard positif sur l'existence, mais, alors que le premier semble être dirigé vers le futur à travers l'optimisme et l'espoir, le second repose davantage sur un bilan positif du passé dont les répercussions se font sentir dans le présent. Dans leur recherche sur les forces des PVVIH âgés, Emlet et al. (2010) dégagent un thème proche de celui de l'attitude positive, à savoir l'optimisme, qu'ils associent à des perspectives positives et un esprit orienté vers le futur. Néanmoins, ils ne relèvent pas de thème analogue à celui du sentiment d'être chanceux qui peut être considéré comme un regard positif sur le passé, lequel contribue à se sentir satisfait de sa situation présente. D'après nos résultats, ce thème serait étroitement relié à ceux des épreuves surmontées et de l'appréciation de la valeur de la vie. Les hommes gais âgés ayant vieilli avec le VIH se considèrent comme privilégiés d'être encore vivants et de vieillir dans des conditions physiques relativement satisfaisantes, au regard de tous les obstacles en lien avec le VIH qu'ils ont dû franchir. Le sentiment d'être chanceux d'avoir survécu au VIH peut donc être considéré comme une force fondamentale dans le processus d'acceptation du vieillissement des participants HARSAH. Compte tenu de l'âgisme et du culte de la jeunesse qui prédomine dans le milieu gai, le fait d' « être heureux d'avoir l'âge qu'ils ont » représente en effet une force exceptionnelle pour les hommes gais âgés vivant avec le VIH.

\section{CONCLUSION}

Pour conclure, l'ensemble de nos résultats démontre la présence de processus de résilience chez les hommes gais âgés vivant avec le VIH. L'originalité de notre étude réside sans doute dans l'attention qui a été portée simultanément aux dimensions sur lesquelles agissent ces processus de résilience mais aussi à leur origine (voir schéma). Il est ainsi apparu que même si les hommes gais âgés doivent faire face à de multiples défis en lien avec l'intersection du VIH, du vieillissement et de l'homosexualité, chacune de ces trois problématiques, et parfois leur conjonction, a permis l'émergence de la résilience qui les aide désormais à composer avec les difficultés de l'existence. 


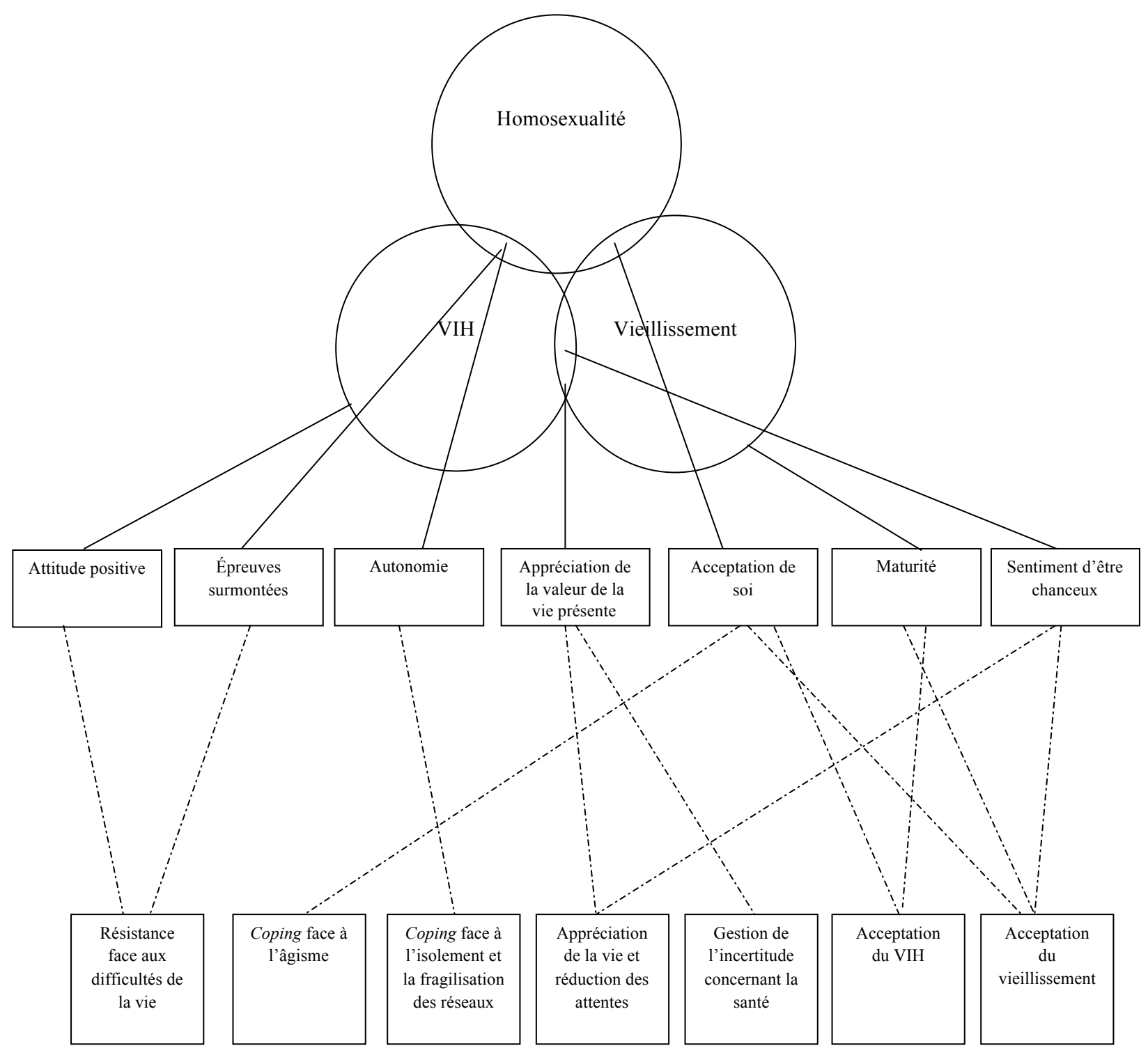

Cette recherche présente néanmoins des limitations, notamment en raison de son petit échantillon. Ainsi, ces résultats de recherche, qui témoignent de l'expérience de 10 HARSAH âgés vivant avec le VIH, demeurent exploratoires et devraient être validés auprès d'un échantillon plus large. En raison de ce petit nombre de participants mais aussi de biais relatifs au lieu du recrutement, nous n'avons pas inclus certaines populations telles que les hommes HARSAH d'origine ethnique ou résidant en milieu rural. Par ailleurs, notre analyse a seulement porté sur l'impact de l'intersection de l'homosexualité, du VIH et du vieillissement, alors que d'autres variables mériteraient d'être prises en considération dans l'analyse, notamment la situation socioéconomique, l'origine ethnique, l'âge et l'état de santé. Enfin, concernant la problématique de la résilience, on peut noter qu'il nous est parfois apparu difficile de distinguer ce qui relevait des forces acquises et ce qui avait trait aux stratégies de coping, les secondes semblant souvent découler des premières. 


\title{
LA RÉSILIENCE DES HOMMES GAIS ÂGÉS VIVANT AVEC LE VIH AU QUÉBEC
}

Nos résultats de recherche laissent cependant émerger des perspectives de recherche à explorer. Il serait notamment intéressant de réaliser une étude comparative sur les forces acquises au fil du parcours de vie avec le VIH et sur les moyens de coping face au vieillissement, en fonction du genre et de l'orientation sexuelle. Une autre piste de recherche concerne la comparaison de l'expérience des HARSAH âgés non utilisateurs de drogues avec celle de ceux ayant été infectés par injection de drogue.

Enfin, il serait important, dans des travaux ultérieurs, de se pencher sur les dimensions de la résilience des HARSAH âgés vivant avec le VIH qui reposent sur les ressources externes et notamment les relations sociales avec l'entourage ou le recours à la spiritualité.

Finalement, cette recherche, bien qu'exploratoire, aura permis de mettre au jour les multiples forces acquises par les HARSAH âgés vivant avec le VIH, à travers leur parcours relié à l'homosexualité, au VIH et au vieillissement. Perçus a priori comme vulnérables, les hommes gais vivant avec le VIH se révèlent donc in fine riches de leurs expériences passées et particulièrement aptes à faire face aux défis du vieillissement.

\section{NOTES}

1. Les termes homme gai, gai, HARSAH (homme ayant des relations sexuelles avec des hommes) sont indifféremment employés pour décrire les participants. L'échantillon n'incluant qu'un homme bisexuel, les passages du texte s'appuyant sur son expérience le désigneront spécifiquement comme tel.

2. Notre traduction de la définition suivante: «Pattern of functioning indicative of positive adaptation in the context of significant risk or adversity ».

3. Le code Hx signifie homme $x$ ans. Ex : H50 signifie homme 50 ans.

\begin{abstract}
The objective of this research is to shed light on the resiliency that older gay men living with HIV developed through their experience of homosexuality, HIV and aging. Following a qualitative approach, this study is based on semi-structured interviews conducted with 10 gay men living with HIV, aged 50 to 71. Data analysis has highlighted 7 themes reflecting the resiliency of this population: maturity, self-acceptance, having overcome difficulties, autonomy, appreciation of the value of life, a positive attitude and the feeling of being fortunate.
\end{abstract}

Keywords: Men, homosexuality, HIV, aging, resiliency, qualitative methodology

\section{RÉFÉRENCES}

Agence de la santé publique du Canada. (2010, juillet). L'infection à VIH et le sida chez les personnes âgées au Canada, Actualités en épidémiologie du VIH/sida, 1-7. Récupéré le 5 octobre 2010 du site : http://www.phac-aspc.gc.ca/ aids-sida/publication/epi/2010/6-fra.php

Applebaum, A. et Brennan, M. (2009). Mental health and depression. Dans M. Brennan, S.E. Karpiak, R.A. Shippy et M.H. Cantor (dir.), Older adults with HIV: An in-depth examination of an emerging population (p. 27-34). New York, NY: Nova Sciences Publishers.

Beasley, M., Thompson, T. et Davidson, J. (2003). Resilience in response to life stress: The effects of coping style and cognitive hardiness. Personality and Individual Differences, 34(1), 77-95.

Butler, S. (2004). Gay, lesbian, bisexual, and transgender (GLBT) elders: The challenges and resilience of this marginalized group. Journal of Human Behavior in the Social Environment, 9(4), 25-44.

Della, B., Wilson, M. et Miller, R.L. (2002). Strategies for managing heterosexism used among African American gay and bisexual men. Journal of Black Psychology, 28(4), 371-391. 
REVUE CANADIENNE DE SANTÉ MENTALE COMMUNAUTAIRE

Denzin, N.K. et Lincoln, Y.S. (1994). Introduction: Entering the field of qualitative research. Dans N.K. Denzin et Y.S. Lincoln (dir.), Handbook of qualitative research (p. 1-17). Thousand Oaks, CA : Sage.

Desquilbet, L., Jacobson, L.P., Fried, L.P., Phair, J.P., Jamieson, B.D., Holloway, M., . . Multicenter AIDS Cohort Study. (2007). HIV-1 infection is associated with an earlier occurrence of a phenotype related to frailty. Journal of Gerontology Series A: Biological Sciences and Medical Sciences, 62(11), 1279-1286.

Effros, R.B., Flechter, C.V., Gebo, K., Halter, J.B., Hazzard, W.R., Horne, F.M. et Huebner R.E. (2008). Aging and infectious diseases: Workshop on HIV infection and aging: What is known and future research directions. Clinical Infectious Disease, 47(4), 542-553.

Emlet, C.A. (2006a). An examination of the social networks and social isolation in older and younger adults living with HIV/AIDS. Health and Social Work, 31(4), 299-308.

Emlet C.A. (2006b). You're awfully old to have this disease: Experiences of stigma and ageism in adults 50 years and older living with HIV/AIDS. The Gerontologist, 46(6), 781-790.

Emlet, C.A., Tozay, S. et Raveis, V.H. (2010). "I'm not going to die from the AIDS": Resilience in aging with HIV disease. The Gerontologist, 51(1), 101-111.

Fredriksen-Goldsen, K.I. et Muraco, A. (2010). Aging and sexual orientation: A 25-year review of the literature. Research on Aging, 32(3), 372-413.

Genke, J. (2004). Resistance and resilience: The untold story of gay men aging with chronic illnesses. Journal of Gay \& Lesbian Social Services, 17(2), 81-95.

Greve, W. et Staudinger, U.M. (2006). Resilience in later adulthood and old age: Resources and potentials for successful aging. Dans D. Cicchetti et D.J. Cohen (dir.), Developmental psychopathology: Vol. 3. Risk, disorder, and adaptation (p. 796-840). Hoboken, NJ : John Wiley \& Sons.

Havlik, R.J. (2009). Health status, comorbidities and health-related quality of life. Dans M. Brennan, S.E. Karpiak, R.A. Shippy et M.H. Cantor (dir.), Older adults with HIV: An in-depth examination of an emerging population (p.13-26), New York, NY: Nova Sciences Publishers.

Herdt, G. et de Vries, B. (dir.). (2004). Gay and lesbian aging: Research and future directions. New York, NY: Springer.

Hildon, Z., Smith, G., Netuveli, G. et Blane, D. (2008). Understanding adversity and resilience at older ages. Social Health III, 30(5), 726-740.

Kalichman, S.C., Heckman, T., Kochman, A. et Bergholte, J. (2000). Depression and thoughts of suicide among middle-aged and older persons living with HIV-AIDS. Psychiatric Services, 51(7), 903-907.

Linsk, N.L. (1997). Experience of older gay and bisexual men living with HIV/AIDS. Journal of Gay, Lesbian, and Bisexual Identity, 2(3/4), 285-308.

Luthar, S.S., Cicchetti, D. et Becker, B. (2000). The construct of resilience: A critical evaluation and guidelines for future work. Child Development, 71(3), 543-562.

Lyons, A., Pitts, M., Grierson, J., Thorpe, R. et Power, J. (2010), Aging with HIV: Health and psychosocial well-being of older gay men. AIDS Care, 22(10), 1236-1244.

Michallet, B. (2009/2010). Résilience : Perspective historique, défis théoriques et enjeux cliniques. Frontières, 22(1/2), $10-18$.

Morrow, D.F. (2001). Older gays and lesbians: Surviving a generation of hate and violence. Journal of Gay \& Lesbian Social Services, 13(1/2), 151-169.

Nichols, J.E., Speer, D.C., Watson, B.J., Watson, M.R., Vergon, T.L., Vallee, C.M. et Meah, J.M. (2002). Aging with HIV: Psychosocial, social and health issues. San Diego, CA : Academic Press.

Ong, A.D., Bergeman, C.S. et Boker, S.M. (2009). Resilience comes of age: Defining features in later adulthood. Journal of Personality, 77(6), 1777-1804.

Pentz, M. (2005). Resilience among older adults with cancer and the importance of social support and spirituality-faith: "I don't have time to die." Journal of Gerontological Work, 44(3/4), 3-22.

Poindexter, C. et Shippy, R.A. (2008). Networks of older New Yorkers with HIV: Fragility, resilience, and transformation. AIDS Patient Care and STDs, 22(9), 723-733.

Power, L., Bell, M. et Freemantle, I. (2010). A National Study of Ageing and HIV (50 plus). Récupéré le 15 avril 2011 du site : http://www.tht.org.uk/binarylibrary/peoplesexperienceofhiv/50-plus-final-report.pdf

Schope, R.D. (2005). Who's afraid of growing old? Gay and lesbian perceptions of aging. Journal of Gerontological Social Work, 45(4), 23-39. 


\section{LA RÉSILIENCE DES HOMMES GAIS ÂGÉS VIVANT AVEC LE VIH AU QUÉBEC}

Shippy, R.A. et Karpiak, S.E. (2005). The aging AHIV/AIDS population: Fragile social networks. Aging \& Mental Health, 9(3), 246-254.

Siegel, K., Raveis, V. et Karus D. (1998). Perceived advantages and disadvantages of age among older HIV-infected adults. Research on Aging, 20 (6), 686-711.

Strauss, A. et Corbin, J. (1998). Basics of qualitative research ( $2^{\circ}$ éd.). Thousand Oaks, CA : Sage.

Vance, D. et Woodley, R.A. (2005). Strengths and distress in adults who are aging with HIV: A pilot study. Psychological Reports, 96(2), 383-386. 